\title{
Synchrotron X-ray Microdiffraction Analysis of Proton Irradiated Polycrystalline Diamond Films
}

\author{
R.L. Newton ${ }^{2 *}$, J.L. Davidson ${ }^{b}$, G. E. Ice ${ }^{c}$, W. Liu ${ }^{c}$ \\ ${ }^{a}$ Marshall Space Flight Center, Huntsville, AL \\ ${ }^{\mathrm{b}}$ Vanderbilt University, Nashville, TN \\ ${ }^{c}$ Oak Ridge National Laboratory, Oak Ridge, TN \\ * Corresponding author email: robby.newton@nasa.gov
}

\begin{abstract}
$\mathrm{X}$-ray microdiffraction is a non-destructive technique that allows for depthresolved, strain measurements with sub-micron spatial resolution. These capabilities make this technique promising for understanding the mechanical properties of MicroElectroMechancial Systems (MEMS). This investigation examined the local strain induced by irradiating a polycrystalline diamond thin film with a dose of $2 \times 10^{17} \mathrm{H}^{+} / \mathrm{cm}^{2}$ protons. Preliminary results indicate that a measurable strain, on the order of $10^{-3}$, was introduced into the film near the End of Range (EOR) region of the protons.
\end{abstract}

\section{Introduction}

Diamond possesses many useful material properties for advanced applications; it is extremely hard, with high wear resistance, chemical inertness, optical transparency, and a wide band-gap. Crystalline diamond has the highest elastic modulus and hardness values known[i]. Polycrystalline diamond produced by techniques such as chemical vapor deposition (CVD) continue to show promise for insertion into advanced technologies such as electronics, Microsystems (MST) and MicroElectroMechanical Systems (MEMS).

The fact that diamond is extremely resistant to radiation damage is an additional very important attribute of this material, especially for space-related applications where high radiation levels are expected. The effects of radiation on the properties of diamond have been studied for decades. However, the majority of these investigations have examined electronic or microstructural changes in monocrystalline diamond, usually using heavy ions $\left[{ }^{\mathrm{ii}}\right]\left[{ }^{\mathrm{iii}}\right]$. Fewer studies have examined the effects of light ions, such as protons, on the microstructural changes of irradiated CVD diamond, particularly as a function of ion implantation depth [ $\left.{ }^{\text {iv }}\right]$. In this paper, we present preliminary results of depth-resolved strain measurement in a heavily proton irradiated CVD diamond film.

$\mathrm{X}$-Ray diffraction, (XRD), is an extremely useful analytical tool for examining the microstructure of materials. It is relatively straightforward, quick, and nondestructive. Additionally, no sample preparation is typically needed. There have been many XRD studies examining CVD diamond and several that have examined CVD diamond using both $\mu$-Raman and XRD studies. ${ }^{v}$, vi, vii However, only in the past few years have the experimental tools been available to allow the XRD study of materials with sub-micron spatial resolution. This has been made possible by the development of ultra-brilliant third-generation synchrotron $\mathrm{x}$-ray sources and advanced $\mathrm{x}$-ray optics. ${ }^{\text {vii }}$ 
Differential-Aperture X-Ray Microscopy (DAXM) is an emerging new method for measuring the local scattering from submicron regions within a sample. In DAXM, subgrain strain measurements are made with submicron spatial resolution in three dimensions. By using charge-coupled device (CCD) area detection, polychromatic x-ray microbeams and computer reconstruction, Laue diffraction patterns are extracted as a function of depth along the penetration direction of the microbeam. ${ }^{\mathrm{ix}}$

Figure 1 presents a schematic of the experimental setup needed to perform DAXM analysis. Strain measurements of less than $10^{-4}$ are possible using this technique. For a detailed example of this procedure, see Larson, et al. ${ }^{\text {ix }}$

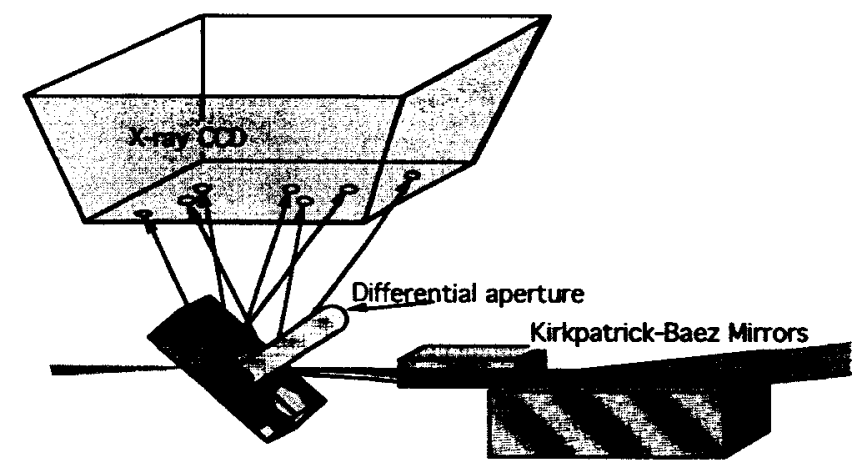

Figure 1. The incident X-ray beam can be selected to have either a monochromatic or a polychromatic spectra. The beam is focused by nondispersive total-external reflection $\mathrm{K}$ $\mathrm{B}$ mirrors onto the sample, which is translated relative to the beam by precision stages. A precision 50-_m-diameter wire is translated near the sample surface to decode the origin of the overlapping Laue patterns. By subtracting CCD images taken before and after small movements of the wire, a pixel by pixel mapping of intensity can be made back to the incident beam path. After scanning across the surface of the sample, a series of single-crystal Laue patterns associated with each location along the beam can be recovered.

\section{Experimental}

Approximately 20 micron thick polycrystalline diamond samples were deposited on 2" single crystal silicon substrate using microwave plasma assisted chemical vapor deposition (MPACVD) at a temperature of $800^{\circ} \mathrm{C}$ and a pressure of 110 Torr. The sample was implanted with protons using a NEC Model 5SDH-2 Pelletron accelerator at ambient temperature. Peltier cooling units were used to prevent overheating of the sample during the irradiation cycle. $600 \mathrm{keV}$ protons were implanted to a dosage of 
$2 \times 10^{17} \mathrm{H}^{+} / \mathrm{cm}^{2}$ at $1.2-6 \times 10^{-7}$ Torr. According to implant modeling software, $600 \mathrm{keV}$ protons implant to a depth of approximately 4 microns. This depth is shown in Figure 2.

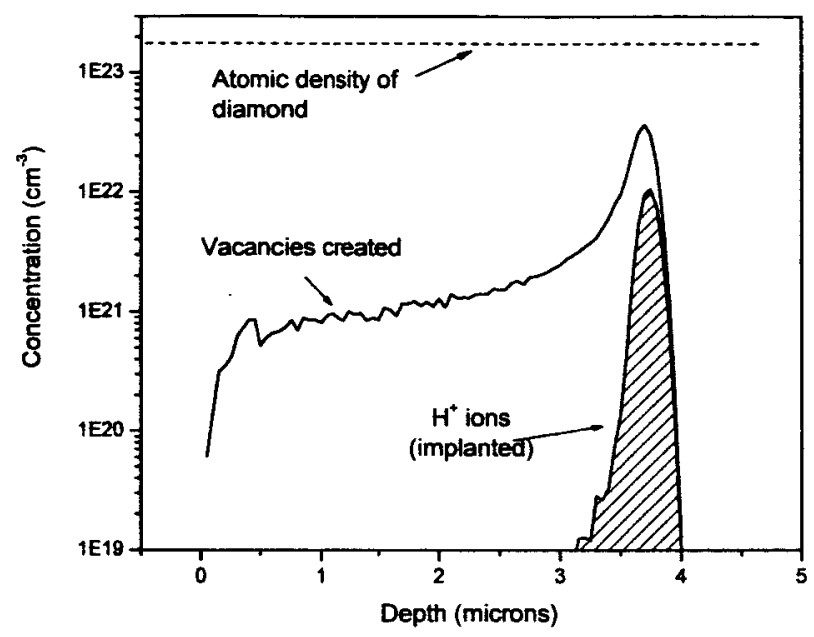

Figure 2. Calculated depth of proton implant into polycrystalline diamond films. The End-of-Range (EOR) is approximately 4 microns.

The sample was not annealed after the irradiation exposure. The $\mu$-X-Ray diffraction measurements were performed on station 34ID-E facility, at the Advanced Photon Source, Argonne National Laboratories. The experimental conditions were as follows: energy range of $8-25 \mathrm{KeV}$ for white beam measurements, Undulator: $12.0 \mathrm{keV}, 4 \mathrm{~mm}$ taper, and $\sim 50$ microrad steering, Micro-beam size: $0.4 \mu \mathrm{m}$ horizontal by $0.5 \mu \mathrm{m}$ vertical.

\section{Results}

The irradiated diamond thin film specimen was examined in detail via depthresolved $\mu$-XRD. This specimen was examined at 6 different locations on the top surface. Both normal strain and shear strain were characterized. Figures 3 and 4 show plots of the normal strain components as a function of depth for a representative location in both the as-deposited and irradiated specimens. Within the range of the proton implantation, the deviatoric strain tensor is positive for the $\square_{\mathrm{x}}$ component, slightly positive for the $\square_{y}$ component, but somewhat negative for the $\square_{z}$ component. Past the EOR, the components return to much lower values and remain fairly constant throughout the depth of the film. The normal strain component $Q_{x x}$ showed the least amount of fluctuation throughout the depth of the film.

Examination of the shear component of the strain tensor indicates fluctuations of all three components, $\square_{x y}, \square_{k y}, \square_{z}$ until reaching a depth of about 8 microns. This is shown in Figures 6 for the heavily irradiated specimen (the as-deposited specimen is shown in Figure 5). Past this depth, all three components return to a very low and consistent value until reaching the end of the film. Unlike the normal strain, the values for $G_{z}$ undulate widely from positive to negative from one micron to the next. This is 
also observed, but to a lesser degree, in the $\square_{z}$ component as well. In both the normal and shear components, the values of the deviatoric strain tensors are on the scale of $10^{-3}$. The $x$-ray microprobe intersected six different grains between the free surface and the silicon substrate, a distance of roughly 20 microns.

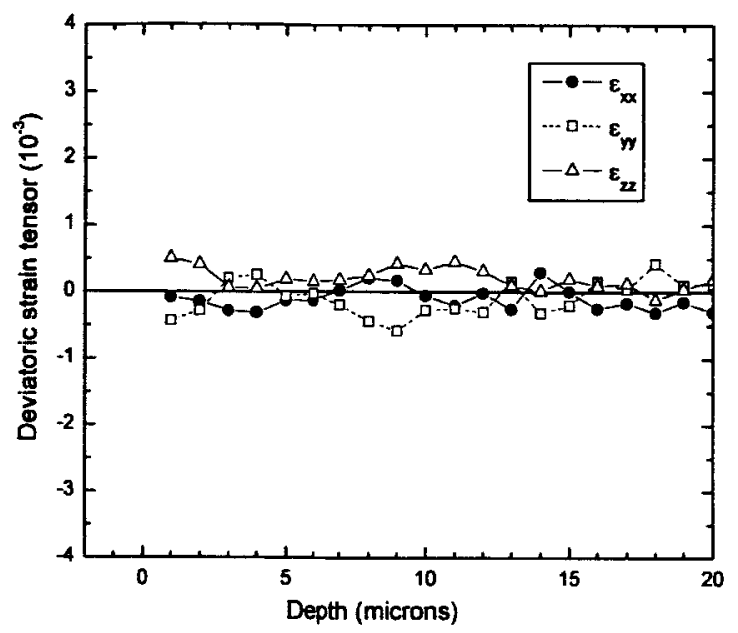

Figure 3. Normal components as a function of depth for the deviatoric strain tensor for the "as-deposited" diamond specimen.

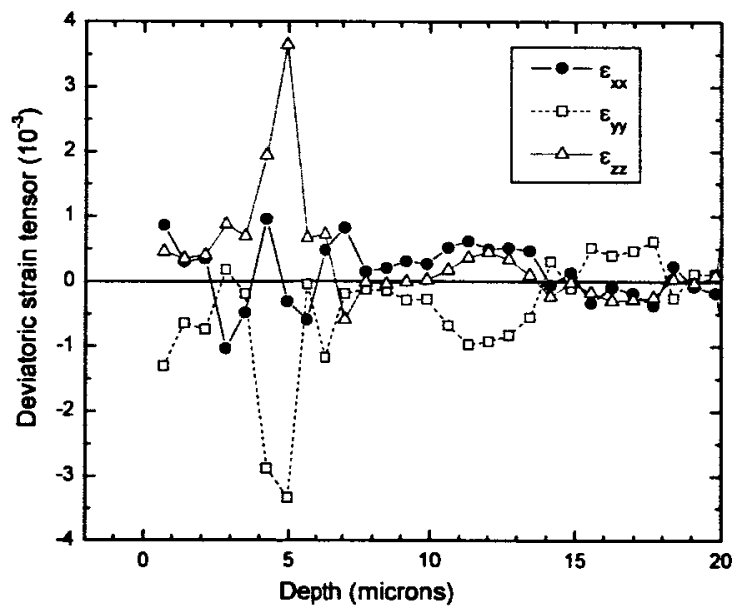

Figure 4. Normal components as a function of depth for the deviatoric strain tensor for the $2 \times 10^{17} \mathrm{H}^{+} / \mathrm{cm}^{2}$ implanted diamond specimen. 


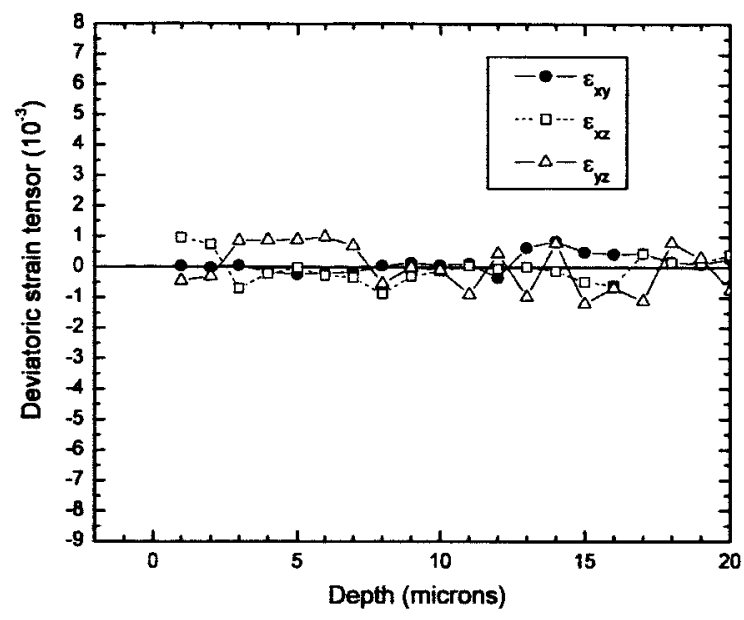

Figure 5. Shear components as a function of depth for the deviatoric strain tensor for the "as-deposited" diamond specimen.

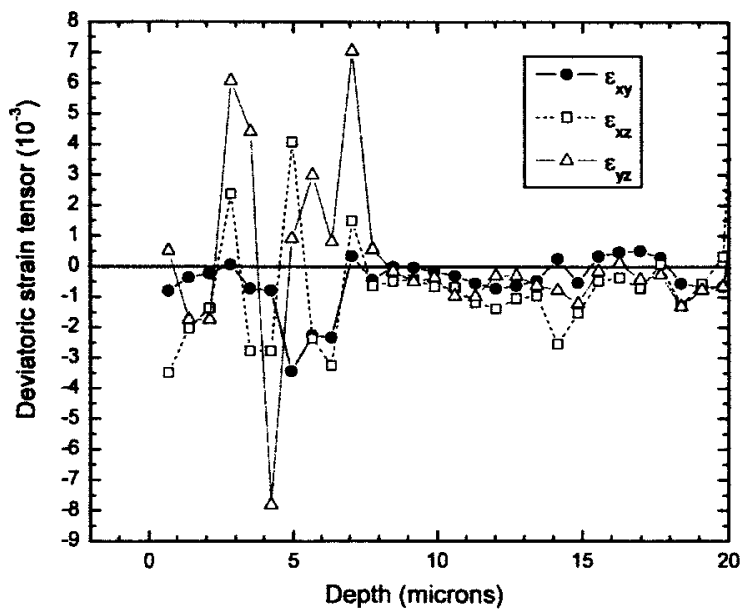

Figure 6. Shear components as a function of depth for the deviatoric strain tensor for the $2 \times 10^{17} \mathrm{H}^{+} / \mathrm{cm}^{2}$ implanted diamond specimen.

\section{Discussion of Results}

Deviatoric Strain Calculations via $\mu$-X-ray Diffraction

The deviatoric strain component of the strain tensor was measured using the $\mu$ XRD system. The strain tensor can be written in terms of both the hydrostatic (dilation) and distortion (deviatoric) components; 


$$
\varepsilon=\left(\begin{array}{ccc}
\varepsilon_{11}-\frac{\Delta}{3} & \varepsilon_{12} & \varepsilon_{13} \\
\varepsilon_{12} & \varepsilon_{22}-\frac{\Delta}{3} & \varepsilon_{23} \\
\varepsilon_{13} & \varepsilon_{23} & \varepsilon_{33}-\frac{\Delta}{3}
\end{array}\right)+\left(\begin{array}{ccc}
\frac{\Delta}{3} & 0 & 0 \\
0 & \frac{\Delta}{3} & 0 \\
0 & 0 & \frac{\Delta}{3}
\end{array}\right),
$$

where $\Delta=\varepsilon_{11}+\varepsilon_{22}+\varepsilon_{33}$. The first term is called the deviatoric strain term and the second is the dilatational strain term ${ }^{\mathrm{x}}$. As was shown in Figures 4 and 5 , the normal and shear components are seen to vary greatly as a function of film depth but the relative values of the tensor elements are small, $\sim 10^{-3}$. For polycrystalline samples, strains are typically assumed to be classified as macro (the average over a region) and micro, fluctuating about the macrostrains due to the elastic inhomogeneity of misoriented crystal grains. As illustrated schematically in Figure 7, the direction for easy deformation changes with crystal orientation.

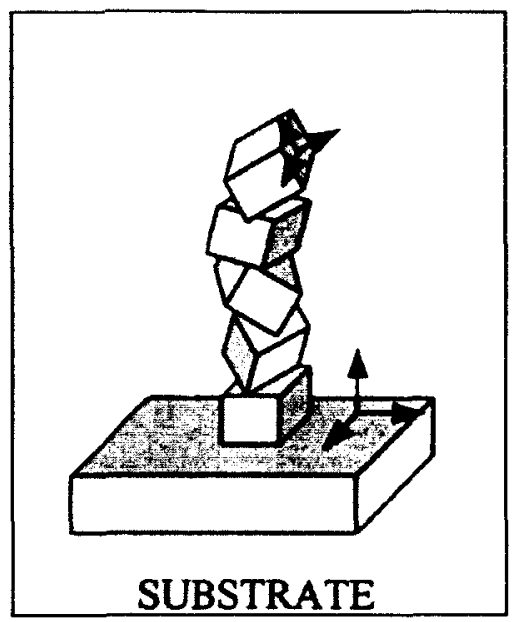

Figure 7. In the polycrystalline state, the crystallites are randomly oriented in three dimensions. ${ }^{\mathrm{xi}}$

In the diamond samples, the grains are mis-oriented, although some degree of texture (columnar growth) was observed in SEM examination. Sub-micron diffraction analysis detects the local strain as a function of location in the material due to grain boundaries and defects present in the film. We note that the local stress can be directly calculated from the measured strain and the crystal compliance matrix. The increase in the values, albeit small, near the implanted region (due to $<100>$ split interstitials created by the implanted protons and confirmed via $\mu$-Raman spectroscopy) indicate that the ion implantation is contained within a narrow region. As a function of depth, the dilatation components change from tensile to compression quickly. This may be attributed to the fact that the $<111>$ direction is the preferred slip direction in diamond and may introduce shear into the crystal. In this case however, sampling methodology is likely contributing 
to these fluctuations in the shear data. This is because the strain measurements are sensitive to peak position fitting and beam optics calibration. As a result, the normal shear components are less sensitive to sample stability during measurement, beam intensity fluctuations, etc. and should be considered more representative of the actual stress states within the films. These $\mu$-XRD results are preliminary and more in-depth analysis will be required to determine conclusively the local $\mu$-strain fields associated with proton implanted polycrystalline diamond.

\section{Conclusions}

In order to study the microstructural effects of proton implantation into diamond films, an experimental method with high spatial resolution was chosen: Differential Aperature $\mu$-X-Ray Microscopy. Using this technique, the deviatoric component of the strain tensor was measured as a function of depth for a $2 \times 10^{17} \mathrm{H}^{+} / \mathrm{cm}^{2}$ implanted specimen. Both the normal and shear components were evaluated. The normal component indicated a positive strain perpendicular to the surface at approximately the EOR for the protons. The creation of a large amount of $<100>$ split interstitial aggregates caused by the proton implant, identified by Raman spectroscopy, plus the addition of the protons themselves creates a dilatory volume change in the region that can account for the observational stress tensor distribution. The maximum strain tensors were on the order of $10^{-3}$.

\section{Acknowledgements}

R.L. Newton supported by NASA's Marshall Space Flight Center. G.E. Ice and W. Liu supported by the Division of Materials Sciences and Engineering U.S. DOE through contract DE-AC05-00OR22725 with the Oak Ridge National Laboratory. The UNICAT facility at the Advanced Photon Source (APS) is supported by the U.S. DOE under Award No. DEFG02-91ER45439, through the Frederick Seitz Materials Research Laboratory at the University of Illinois at Urbana-Champaign, the Oak Ridge National Laboratory (U.S. DOE contract DE-AC05-00OR22725 with UT-Battelle LLC) and UOP LLC. The APS is supported by the U.S. DOE, Basic Energy Sciences, Office of Science under contract No. W-31-09-ENG-38."

\section{References}

i J.P. Sullivan, T.A. Friedmann, and K. Hjoit, MRS Bulletin, April, (2001) 309.

ii D.N. Jamieson, S. Prawer, K.W. Nugent, and S.P. Dooley, Nucl. Instr. Meth. B106, (1995) 641. 
iii J.O. Orwa, K.W. Nugent, , D.N. Jamieson, and S. Prawer, Phys. Rev. B62, (2000) 5461.

${ }^{i v}$ A.A. Gippius, R.A. Khmelnitsky, V.A. Dravin, and A.V. Khomich, Physica B 308-310, (2001) 573.

v. Durand, O, Olivier, J, Bisaro, R., Galtier, P., Kruger, J.K., Brierley, C.J., and Kennedy, G.R., Appl. Phys. Letters, 75 (13), 1881 (1999).

vi. Mohrbacher, H, Van Acker, K., Blanpain, B., Van Houtte, P., and Celis, J-P., J. Mat. Res. 111 (7), 1776 (1996).

vii. Pandley, M., J of Alloys and Compounds, 333, 260-265 (2002).

viii. Chung, J.-S., and Ice, G. E., J. of Appl. Phys. 86 (9), 5249 (1999).

ix. Larson, B.C., Yang, W., Ice, G.E., Budal, J.D., and Tischler, J.Z., Nature, 415, 887 (2002).

x. Chung, J.S., and Ice, G.E., J. Applied Physics, 86, 9, 5249 (1999).

xi. Anastassakis, E., and Siakavellas, M., J of Applied Physics, 90, 1, 144 (2001). 